\title{
Effect of Implementing Nursing Care on Respiratory Parameters for Patients with Acute Exacerbation of Chronic Obstructive Pulmonary Diseases.
}

\author{
Azza Salman Ahmed ${ }^{1}$, Amany Omar Mohamed ${ }^{2}$, Salah Ahmed Mohammed ${ }^{3}$, Mervat Anwer AbdEl -Aziz ${ }^{4}$ \& \\ Mogedda Mohamed Mehany ${ }^{5}$. \\ 1. Master degree in Critical Care Nursing at Sohage University Hospital, Egypt. \\ 2. Prof. Dr. of intrinsic medicine, Faculty of Medicine, Assuit University, Egypt. \\ 3. Prof.Dr.in Anesthia \& ICU Department,faculty of medicine, Sohage University, Egypt. \\ 4. Assistant professor of Critical Care \& emergency nursing, Faculty of Nursing, Assuit University, Egypt. \\ 5. Assistant professor of Critical Care \& emergency nursing, Faculty of Nursing, Assuit University, Egypt.
}

\begin{abstract}
Acute exacerbation usually accompanied by increased cough, fever, wheezing, nursing care have been involved in delivering non-pharmacological interventions aimed to reducing symptoms Aim of this study was to evaluate effect of implementing nursing care on respiratory parameters for patients with acute exacerbation of chronic obstructive pulmonary diseases. Design: A quasi experimental research design was used to conduct this research. A convenient sample consisted of 60 patients, they were enrolled into study group $(n=30)$ and control group $(n=30)$ who were admitted to Intensive care unit at Sohag university hospital \& chest care unit at Assiut University hospital Tool: this study comprised from Tool I: patient's demographic and medical data. Tool II: Patient's clinical assessment sheet consists from: Part 1: Assessment of homodynamic parameters Part II: Respiratory parameters. Part III: laboratory investigation and Receiving medications. The main results: result revealed that there is significant difference regard to temperature in study group compared with control group in $1^{\text {st }} \& 4^{\text {rd }}$ days. As regard respiratory parameters the result revealed significant difference regarding heart rate at $4^{\text {rd }} \& 7^{\text {th }}$ day. In addition current study revealed that highly significant difference in both groups regard breath sound $(\mathbf{p}<\mathbf{0 . 0 0 5})$. Conclusion: Efficient nursing management after acute exacerbation of chronic obstructive pulmonary disease improves respiratory parameters.
\end{abstract}

\section{Keywords: Acute Exacerbation COPD, Respiratory Parameters \& Nursing Care.}

\section{Introduction}

exacerbation of chronic obstructive pulmonary disease is an acute event characterized by sustained worsening of any of the patient's respiratory symptoms (cough, sputum quantity and/or character, dyspnoea) that is beyond normal day-to-day variation and leads to a change in medication, and where other causes of acute breathlessness have been clinically excluded (Dheeraj et al ., 2014).

Exacerbations of chronic obstructive pulmonary disease (COPD) are common and have a profound influence on the health status of patients. Repeated exacerbations are associated with increased mortality, reduced quality of life, and accelerated decline in lung function Moreover, exacerbations of COPD cause frequent and prolonged hospital admissions, conditional decline, and repeated exacerbations (Williams et al., 2011).

Nursing care include (bronchodilators, corticosteroids, antibiotics and supplemental oxygen should also be initiated and titrated to achieve an oxygen saturation of $88 \%$ to $92 \%$. As an alternative to oxygen therapy, oxygen via high-flow nasal cannula or noninvasive positive pressure ventilation can also be used to improve oxygenation and ventilation and decrease hypercapnia in acute hypoxemic respiratory failure (Global Initiative for chronic obstructive lung disease. 2018).

The diagnosis of an exacerbation is primarily clinical, and is based upon of sudden change of symptoms (baseline dyspnea, cough, and/or sputum production) that is beyond normal day-to-day variation. Worsening breathlessness is the cardinal symptom of an exacerbation and is usually accompanied by increased cough, fever, wheezing, chest tightness, and change in the color and/or volume of sputum. There may also be non-specific manifestations, such as tachycardia, tachypnea, fever, malaise, insomnia, sleepiness, fatigue, depression, and confusion; these are more common in the elderly (Roberts et al., 2017).

Criteria for patients who are hypoxemic during an exacerbation; arterial blood gases and/or pulseoximetry should be evaluated prior to hospital discharge and in the following 3 months. If the patient remains hypoxemic, long-term supplemental oxygen therapy may be required. Also, patients should be given clear instructions about when and how to stop their corticosteroid treatment (Global Initiative for Chronic Obstructive Lung Disease, 2018). 
Oxygen therapy is important to prevent severe hypoxemia during exacerbations of COPD, but high levels of supplemental oxygen may contribute to hypercapnia, acidosis and death. Oxygen therapy should be titrated to achieve oxyhaemoglobin saturation ( $\mathrm{SpO} 2)$ between 88 and 92\%, 31 and is usually administered via nasal prongs or a venture mask. Physiotherapists frequently use breathing exercises to relieve dyspnea, improve thoracicabdominal co-ordination and enhance functional capacity in people with acute exacerbations of COPD (Holland, 2014).

A major advance in nursing care for acute exacerbations of COPD has been the implementation of noninvasive positive pressure ventilation (NIV). NIV has been shown to improve acute respiratory acidosis (increases $\mathrm{pH}$ and decreases partial pressure of arterial carbon dioxide), decrease respiratory rate, work of breathing, severity of breathlessness, complications such as ventilator associated pneumonia and length of stay. Not only can intubations be avoided, but mortality for severe COPD exacerbations is also substantially reduced. A recent randomized trial testing the benefit of a helium oxygen mixture for use in noninvasive ventilator support in COPD exacerbations did not show superiority (WHO, 2013).

\section{Significance of the study}

Chronic obstructive pulmonary disease (COPD) prevalence, morbidity and mortality vary across countries. In the USA, COPD affects approximately 24 million Americans, results in about 120,000 deaths a year and is now the third leading cause of death [Kochanek et al. 2011], chronic lower respiratory disease, composed chiefly of chronic obstructive pulmonary disease (COPD), was the third leading cause of death in the United States Kochanek, (2014). Therefore, Statistics of Egyptian Intensive care unit at Assuit University Hospital admitted to Chest care unit is ranged 500 patients in the years of 2016. While in Sohag university hospital in the years of (2015-2016). Revealed that the number of patient admitted Intensive care unit were (50) patients (Hospital records of patients Sohag University).

\section{Aim of the study}

Evaluate effect implementing nursing care on respiratory parameters for patients with acute exacerbation of chronic pulmonary disease.

\section{Patients and methods}

\section{Design}

A quasi experimental research design was adopted to conduct this study.
Hypotheses: To fulfill the aim of the study the following research hypothesis were formulated:-

Hypotheses (1) acute exacerbation COPD patients who are exposed to implementing nursing care will exhibit better mean score of hemodynamic parameters than of the control group

Hypotheses (2) acute exacerbation COPD patients who are exposed to implementing nursing care will exhibit better mean score of respiratory parameters than of the control group

\section{Technical design}

\section{Setting}

The study was conducted at the (ICU) unit at Sohag university hospital \& Chest care unit at Assuit university hospital.

\section{Sample}

A purposive sample consisted of 60 critically ill adult patients (male and female) who were admitted to Intensive care unit at shag university hospital \& chest care unit at Assiut University hospital They were matched \& randomly assigned into two equally groups 30 patient (control group \& study group) each considering the following:-

\section{Criteria of sample selection:-}

\section{Inclusion criteria:}

* Diagnosed as acute exacerbation of chronic obstructive pulmonary disease.

* Recently admitted within 24hours.

* Both gender (males and females).

Exclusion criteria

* Severe head injury, increase intracranial pressure.

* Terminal diseases or inoperable cancer

* Autoimmune diseases.

* Age more than 60 years.

Tools

Two main tools were developed by the researcher and used in this study. The tool was revised by a panel of 5 nursing and medical experts, and tested then piloted by the investigator.

Tool one: patient's demographic data and medical data: this tool was developed by the researcher after reviewing the related literature's to assess patient's demographic data and health relevant data it comprised two parts.

Part I: Socio- demographic data.

This includes patient's age, gender, date of admission and date of discharge.

\section{Part II: Medical data}

Which includes diagnosis, patient complain (productive cough, chest wheezing, dyspnea and fever. 
Tool two: Patient's clinical assessment sheet "this tool consists of two main parts as following Part 1: Homodynamic parameters assessment

This was developed by the researcher to evaluate the patient homodynamic statute and study the effect of nursing care to improve its parameter such as (Temperature $(\mathrm{C})$, Heart rate (HR), Blood pressure $(\mathrm{BP})$, Central veins pressure(CVP) readings and Mean arterial pressure (MAP).

Part II: Respiratory parameters assessment it included:

(Respiratory rate, breath sound as normal, wheeze, crackles, crepitating) and arterial blood gases are monitoring (ABG): for (PH, Pao2, Paco2, $\mathrm{Hco} 3$ and Sao2).

Part III: A- Laboratory investigations that included: complete blood picture as (white blood cell, blood hemoglobuline level, red blood cell and prothrombine time)

B- Medications assessment and monitoring.

\section{Methods}

This study where carried out through three main phases as following

\section{1- The preparatory phase}

- An official Permission to conduct the study was obtained from the hospital responsible authorities in the General Intensive Care Unit at Sohag university hospital \& chest care unit at Assuit University Hospital after explaining the aim and nature of the study.

- Content validity: The tools were tested for content related validity by jury of 6 specialists in the field of critical care nursing and critical care medicine from Shag University \& chest care unit at Assuit University Hospital and the necessary modification were done.

- A pilot study carried out before starting of data collection to test the applicability and clarity of the study tools on $10 \%$ the study sample, The purpose of the pilot study was: to ascertain the relevance of the tool, detect any problem might interfere with the process of data collection and to estimate the time needed to complete the study, define the modification required in the tool used, and the necessary modification was done prior to data collection, The studied subjects were excluded from the actual study.

- An approval was obtained from the local ethical committee and the study was followed the common ethical principles in clinical research.

- The tool used in this study was developed based on reviewing the relevant literature.

- Protection of human rights (ethical considerations): Informed consent was obtained from each patient or from the responsible person for the unconscious patients. The investigator emphasized that the participation is voluntary and the confidentiality and anonymity of the subjects were assured through coding the data. Subjects were assured that can they withdraw from the study at any time without any rational.

- Implementation phase Purpose of the study was simply explained to patients and their relatives in case of unconsciousness.

- The researcher started to collect data from patients on first day and once per shift for seven days of admission.

- The study involved 60 patients who admitted to the General Intensive Care Unit at Sohag University hospital \& chest care unit at Assuit University Hospital over a period of 10 months

- Starting from April 2017 until April 2018. In addition, the following data were collected on first day from patients and from patients records tools.

The control group

They received the routine hospital care for critically ill patients without intervention from the researcher.

- The study group

The patient received the developed nursing care for acute exacerbation of chronic obstructive pulmonary disease $\&$ the intervention group was exposed to the following intervention from the first day of admission until the seventh day of the study during the different three shifts. The researcher assessed critically ill patients of (AECOPD) by used tool two.

Implement the chest physiotherapy procedure for study group.

- Each patient was placed in baseline semi setting position with elevated head of the bed 30 degree for 10 minutes before commencement of study.

- The measurement of hemodynamic parameters, blood gases and auscultator finding were done to determine breath sounds or any abnormal sounds before starting and after 2 hours of chest physiotherapy.

- Each postural drainage position were maintained for 5 minute and vibration followed by suction.

- Postural drainage positions were modified according to the patient's condition.

- Percussion and vibration were applied over the lung, commonly accepted anatomical landmarks for percussion and vibration includes $10^{\text {th }}$ thoracic vertebra posteriorly and the xiphiod an anteriorly with normal respiration.

- Percussion and vibration was avoided over the kidney, spinal column, breasts, floating rib, scapula and sternum. 
- Oxygen saturation and heart rate was monitoring using pulse Oximetry

- Suction was done with a sterile technique. Patient was pre-oxygenated with $100 \%$ before suction.

- A suction procured was accomplished before getting in postural drainage and following percussion and vibration the suction time less than 10 seconds to prevent desaturation.

- During turning the patient any tubes and connection attached to the patient was observed as ECG monitor, feeding tube, urinary catheter, arterial line and central venous pressure line to avoid pulling, stretching or kilning these tube were avoided.

- The bronchodilator treatments prescribed by physical were given 15-30 minutes before chest physiotherapy.

- At the end of the procedure leaving the patient to the routine nursing intervention.

\section{Evaluation phase}

Both groups were evaluated daily during the three shifts using tool two ( $1^{\text {st }}$ part and $2^{\text {nd }}$ part $)$ vital signs (temperature, pulse and BP) were assessed every 2 hours of chest physiotherapy.

\section{Results}

Table (1): Comparison between the study \& control group as regard patients demographic data.

\begin{tabular}{|c|c|c|c|c|c|}
\hline Demographic data & \multicolumn{2}{|c|}{$\begin{array}{c}\text { Study group } \\
\mathbf{N}=\mathbf{3 0}\end{array}$} & \multicolumn{2}{|c|}{$\begin{array}{c}\text { Control group } \\
\mathbf{N}=\mathbf{3 0}\end{array}$} & \multirow[t]{2}{*}{$P$ value } \\
\hline Age "ys" & $\mathbf{N}$ & $\%$ & $\mathbf{N}$ & $\%$ & \\
\hline Mean \pm S.D & \multicolumn{2}{|c|}{$53.63 \pm 7.425$} & \multicolumn{2}{|c|}{$51.93 \pm 8.034$} & \multirow{5}{*}{$0.913 \mathrm{~ns}$} \\
\hline $30<40$ & 1 & 3.3 & 2 & 6.7 & \\
\hline $40<50$ & 5 & 16.7 & 5 & 16.7 & \\
\hline $50<60$ & 16 & 53.3 & 14 & 46.7 & \\
\hline More than 60 & 8 & 26.7 & 9 & 30.0 & \\
\hline \multicolumn{6}{|l|}{ Gender } \\
\hline Male & 21 & 70.0 & 21 & 70.0 & \multirow[t]{2}{*}{$0.611 \mathrm{~ns}$} \\
\hline Female & 9 & 30.0 & 9 & 30.0 & \\
\hline
\end{tabular}

n.s $p>0.05$ no significance $\quad * p<0.05$ significance $\quad * * p<0.001$ moderate significance $* * * p<0.000$ high significance
Respiratory parameters as: (Respiratory rate, breath sound as normal, wheeze, crackles) were assessed every 2 hours of chest physiotherapy.

Laboratory investigations such as ABGs were done daily from $1^{\text {st }}$ day of admission and until the seven day of the study. Total leukocytes count and serum hemoglobin was done at the time of admission and repeated at the $4^{\text {th }}, 7^{\text {th }}$ day and when needed.

Medication monitoring and assessment daily during the three shifts and to determine the effect of implementing nursing care on respiratory parameters for patients with acute exacerbation of chronic obstructive disease.

\section{Statistical analysis}

The collected data were coded entered to SPSS 20.0 statistical software package and then analysis. The data presented using frequencies and percentages descriptive statistics in the form of for qualitative variables, while means and standard deviations used for quantitative variables. Chi-square test was used for non-parametric data to determine significant. Quantitative continuous data were compared using analysis of variance test in case of comparisons between two independent groups. Tests of significance were considered as follows: $\mathrm{P}>0.05$ non-significant $* \mathrm{P}<0.05 \quad$ significant $\quad * * \mathrm{P}<0.01$ moderate significant $* * * \mathrm{P}<0.001$ highly significant. 
Table (2): Comparison between the study \& control group as regard medical data.

\begin{tabular}{|c|c|c|c|c|c|}
\hline \multirow{2}{*}{$\begin{array}{l}\text { Medical data } \\
\text { Pt. complain }\end{array}$} & \multicolumn{2}{|c|}{$\begin{array}{c}\text { Study group } \\
\mathbf{N}=\mathbf{3 0}\end{array}$} & \multicolumn{2}{|c|}{$\begin{array}{c}\text { Control group } \\
\mathbf{N}=\mathbf{3 0}\end{array}$} & \multirow[t]{2}{*}{$P$ value } \\
\hline & $\mathrm{N}$ & $\%$ & $\mathrm{~N}$ & $\%$ & \\
\hline productive cough & 8 & 26.7 & 10 & 33.3 & \multirow{4}{*}{0.603} \\
\hline chest wheezing & 5 & 16.7 & 8 & 26.7 & \\
\hline Fever \& chest pain & 9 & 30.0 & 7 & 23.3 & \\
\hline Dyspnea & 8 & 26.7 & 5 & 16.7 & \\
\hline \multicolumn{6}{|c|}{ 2-Presence of any chronic disease. } \\
\hline Hepatic disease & 7 & 23.3 & 16 & 53.3 & $0.016^{*}$ \\
\hline Stroke & 1 & 3.3 & 1 & 3.3 & $0.754 \mathrm{~ns}$ \\
\hline Kidney disease & 5 & 16.7 & 6 & 20.0 & $0.500 \mathrm{~ns}$ \\
\hline Ischemic disorder & 8 & 26.7 & 8 & 26.7 & $0.614 \mathrm{~ns}$ \\
\hline Hypertensive & 6 & 20.0 & 6 & 20.0 & $0.626 \mathrm{~ns}$ \\
\hline D.M & 12 & 40.0 & 10 & 33.3 & $0.395 \mathrm{~ns}$ \\
\hline \multicolumn{6}{|l|}{ Past medical history } \\
\hline Yes & 12 & 40.0 & 20 & 66.7 & \multirow{2}{*}{$0.038 *$} \\
\hline No & 18 & 60.0 & 10 & 33.33 & \\
\hline
\end{tabular}

n.s $p>0.05$ no significance $\quad * p<0.05$ significance $\quad * * p<0.001$ moderate significance

$* * * p<0.000$ high significance

Table (3): Comparison between the study \& control groups as regard to hematological laboratory investigation\& mean values in both groups.

\begin{tabular}{|c|c|c|c|c|c|}
\hline \multirow{2}{*}{\multicolumn{2}{|c|}{ Laboratory investigation }} & \multirow[t]{2}{*}{ Days } & $\begin{array}{c}\text { Study group } \\
\mathbf{N}=\mathbf{3 0}\end{array}$ & $\begin{array}{c}\text { Control group } \\
\mathrm{N}=\mathbf{3 0}\end{array}$ & \multirow[t]{2}{*}{$P$ value } \\
\hline & & & Mean \pm S.D & Mean \pm S.D & \\
\hline \multirow{9}{*}{ CBC } & \multirow[t]{3}{*}{ WBCS } & $\mathbf{1}^{\mathrm{st}}$ & $1.53 \pm 9.56$ & $5.57 \pm 112.57$ & 0.315 \\
\hline & & $4^{\text {rd }}$ & $14.34 \pm 5.88$ & $16.6 \pm 10.46$ & 0.307 \\
\hline & & $7^{\text {th }}$ & $14.97 \pm 6.36$ & $14.27 \pm 7.43$ & 0.694 \\
\hline & \multirow[t]{3}{*}{$\mathbf{H b}$} & $1^{\mathrm{st}}$ & $12.18 \pm 2.97$ & $12.6 \pm 2.37$ & 0.610 \\
\hline & & $4^{\mathrm{rd}}$ & $1.18 \pm 2.32$ & $1.16 \pm 1.78$ & 0.697 \\
\hline & & $7^{\text {th }}$ & $1.15 \pm 1.78$ & $1.18 \pm 1.72$ & 0.365 \\
\hline & \multirow[t]{3}{*}{ RBCS } & $1^{\text {st }}$ & $4.69 \pm 1.08$ & $5.089 \pm .690$ & 0.095 \\
\hline & & $4^{\text {rd }}$ & $4.68 \pm .676$ & $4.87 \pm .619$ & 0.263 \\
\hline & & $7^{\text {th }}$ & $4.79 \pm .641$ & $4.82 \pm .697$ & 0.874 \\
\hline \multirow{3}{*}{$\begin{array}{l}\text { Blood Coagulation } \\
\text { test }\end{array}$} & \multirow{3}{*}{$\begin{array}{l}\text { Prothrombin } \\
\text { time }\end{array}$} & $1^{\mathrm{st}}$ & $15.13 \pm 4.57$ & $15.87 \pm 4.71$ & 0.540 \\
\hline & & $4^{\text {rd }}$ & $1.49 \pm 3.42$ & $1.44 \pm 2.23$ & 0.521 \\
\hline & & $7^{\text {th }}$ & $20.56 \pm 34.61$ & $14.48 \pm 1.97$ & 0.341 \\
\hline
\end{tabular}

n.s $p>0.05$ no significance $* p<0.05$ significance $* * p<0.001$ moderate significance $* * * p<0.000$ high significance

Table (4): Comparison between both study \& control groups in relation to receiving medications.

\begin{tabular}{|c|c|c|c|c|c|c|}
\hline \multirow[t]{2}{*}{ Medications } & \multirow[t]{2}{*}{ Days } & \multicolumn{2}{|c|}{$\begin{array}{c}\text { Study group } \\
\mathbf{N}=\mathbf{3 0}\end{array}$} & \multicolumn{2}{|c|}{$\begin{array}{c}\text { Control group } \\
\mathbf{N}=\mathbf{3 0}\end{array}$} & \multirow[t]{2}{*}{$P$ value } \\
\hline & & $\mathbf{N}$ & $\%$ & $\mathbf{N}$ & $\%$ & \\
\hline \multirow[t]{3}{*}{ Anticoagulant } & $1^{\mathrm{st}}$ & 10 & 33.3 & 13 & 433.3 & 0.434 \\
\hline & $4^{\text {th }}$ & 10 & 33.3 & 17 & 56.6 & 0.07 \\
\hline & $7^{\text {th }}$ & 9 & 30.0 & 13 & 43.3 & 0.292 \\
\hline \multirow[t]{3}{*}{ Antihypertensive } & $1^{\mathrm{st}}$ & 8 & 26.6 & 9 & 30.0 & 0.779 \\
\hline & $4^{\text {th }}$ & 10 & 33.3 & 9 & 30.0 & 0.786 \\
\hline & $7^{\text {th }}$ & 10 & 33.3 & 9 & 30.0 & 0.786 \\
\hline
\end{tabular}




\begin{tabular}{|c|c|c|c|c|c|c|}
\hline \multirow[t]{2}{*}{ Medications } & \multirow{2}{*}{ Days } & \multicolumn{2}{|c|}{$\begin{array}{c}\text { Study group } \\
\mathbf{N}=\mathbf{3 0}\end{array}$} & \multicolumn{2}{|c|}{$\begin{array}{c}\text { Control group } \\
\mathbf{N}=\mathbf{3 0}\end{array}$} & \multirow[t]{2}{*}{$P$ value } \\
\hline & & $\mathbf{N}$ & $\%$ & $\mathbf{N}$ & $\%$ & \\
\hline \multirow[t]{3}{*}{ Osmotic diuretic } & $1^{\text {st }}$ & 11 & 36.6 & 11 & 36.6 & 1.000 \\
\hline & $4^{\text {th }}$ & 10 & 33.3 & 11 & 36.6 & 0.791 \\
\hline & $7^{\text {th }}$ & 11 & 36.6 & 10 & 33.3 & 0.791 \\
\hline \multirow[t]{3}{*}{ Antibiotic } & $1^{\text {st }}$ & 30 & 100.0 & 30 & 100.0 & 1.000 \\
\hline & $4^{\text {th }}$ & 30 & 100.0 & 29 & 96.6 & 0.321 \\
\hline & $7^{\text {th }}$ & 30 & 100.0 & 30 & 100.0 & 1.000 \\
\hline \multirow[t]{3}{*}{ Bronchodilators } & $1^{\text {st }}$ & 22 & 73.3 & 28 & 93.3 & $0.038^{*}$ \\
\hline & $4^{\text {th }}$ & 24 & 80.0 & 26 & 86.6 & 0.768 \\
\hline & $7^{\text {th }}$ & 23 & 76.6 & 26 & 86.6 & 0.325 \\
\hline \multirow{3}{*}{ Antipyretic } & $1^{\text {st }}$ & 7 & 23.3 & 10 & 33.3 & 0.399 \\
\hline & $4^{\text {th }}$ & 3 & 10.0 & 8 & 26.6 & 0.098 \\
\hline & $7^{\text {th }}$ & 3 & 10.0 & 7 & 23.3 & 0.171 \\
\hline \multirow[t]{3}{*}{ Insulin } & $1^{\text {st }}$ & 13 & 43.3 & 11 & 36.6 & 0.605 \\
\hline & $4^{\text {th }}$ & 11 & 36.6 & 11 & 36.6 & 0.324 \\
\hline & $7^{\text {th }}$ & 11 & 36.6 & 9 & 30.0 & 0.625 \\
\hline \multirow[t]{3}{*}{ Corticosteroid } & $1^{\text {st }}$ & 29 & 96.6 & 23 & 76.6 & $0.023^{*}$ \\
\hline & $4^{\text {th }}$ & 26 & 86.6 & 24 & 80.0 & 0.497 \\
\hline & $7^{\text {th }}$ & 29 & 96.6 & 25 & 83.3 & 0.088 \\
\hline \multirow[t]{3}{*}{ Inhalation } & $1^{\text {st }}$ & 30 & 100.0 & 28 & 93.3 & 0.241 \\
\hline & $4^{\text {th }}$ & 30 & 100.0 & 29 & 96.6 & 0.321 \\
\hline & $7^{\text {th }}$ & 30 & 100.0 & 30 & 100.0 & ---- \\
\hline
\end{tabular}

Table (5): Comparison between the study and control groups in relation to hemodynamic parameter \& mean values in both groups.

\begin{tabular}{|c|c|c|c|c|c|c|}
\hline \multirow{2}{*}{$\begin{array}{c}\text { Hemodynamic } \\
\text { parameters }\end{array}$} & \multicolumn{2}{|c|}{ (base line) before CPT } & \multirow[b]{2}{*}{$p$ value } & \multicolumn{2}{|c|}{ After 2h of CPT } & \multirow[b]{2}{*}{ p value } \\
\hline & $\begin{array}{l}\text { Study } \\
\text { group }\end{array}$ & $\begin{array}{l}\text { Control } \\
\text { group }\end{array}$ & & $\begin{array}{l}\text { study } \\
\text { group }\end{array}$ & control group & \\
\hline$\overline{T e m} 1^{\text {st }}$ day & $37.9 \pm 0.5$ & $38.1 \pm 0.61$ & 0.097 & $37.7 \pm 0.48$ & $37.8 \pm 0.54$ & 0.254 \\
\hline $4^{\text {rd }}$ day & $37.8 \pm 0.50$ & $37.9 \pm 0.53$ & 0.469 & $36.4 \pm 6.33$ & $37.8 \pm 0.46$ & 0.233 \\
\hline $7^{\text {th }}$ day & $37.7 \pm 0.43$ & $38.05 \pm 0.48$ & $0.005 * *$ & $37.4 \pm 0.28$ & $37.9 \pm 0.48$ & $0.000 * * *$ \\
\hline Pulse $1^{\text {st }}$ day & $98.32 \pm 15.7$ & $98.32 \pm 11.39$ & $0.004^{*}$ & $98.36 \pm 14.79$ & $98.36 \pm 12.1$ & 0.008 \\
\hline $4^{\text {rd }}$ day & $101.80 \pm 12.47$ & $98.62 \pm 13.82$ & 0.353 & $101.30 \pm 12.62$ & $98.33 \pm 12.70$ & 0.368 \\
\hline $7^{\text {th }}$ day & $95.088 \pm 12.40$ & $105.53 \pm 12.85$ & $0.002 * *$ & $95.088 \pm 12.40$ & $105.53 \pm 12.85$ & $0.002 * *$ \\
\hline 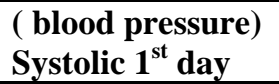 & $119.93 \pm 16.59$ & $117.14 \pm 15.28$ & 0.501 & $123.80 \pm 17.64$ & $119.84 \pm 14.13$ & 0.346 \\
\hline $4^{\text {rd }}$ day & $120.10 \pm 15.15$ & $116.50 \pm 16.34$ & 0.380 & $117.26 \pm 15.42$ & $116.44 \pm 17.15$ & 0.848 \\
\hline $7^{\text {th }}$ day & $115.30 \pm 14.35$ & $110.70 \pm 22.05$ & 0.341 & $115.85 \pm 14.17$ & $109.90 \pm 19.81$ & 0.271 \\
\hline Diastolic & $71.49 \pm 8.46$ & $71.67 \pm 10.19$ & 0.942 & $73.27 \pm 9.49$ & $71.78 \pm 9.34$ & 0.542 \\
\hline $4^{\text {rd }}$ day & $71.70 \pm 9.132$ & $71.12 \pm 13.25$ & 0.842 & $70.54 \pm 8.82$ & $0.67 \pm 11.32$ & 0.960 \\
\hline $7^{\text {th }}$ day & $70.12 \pm 7.95$ & $66.95 \pm 15.41$ & 0.321 & $69.89 \pm 7.99$ & $66.12 \pm 13.65$ & 0.196 \\
\hline${\text { MAP } 1^{\text {st }} \text { day }}$ & $40.15 \pm 5.48$ & $38.86 \pm 4.79$ & 0.444 & $40.63 \pm 5.64$ & $39.27 \pm 4.57$ & 0.311 \\
\hline $4^{\text {rd }}$ day & $39.20 \pm 4.95$ & $38.67 \pm 5.38$ & 0.349 & $38.78 \pm 5.20$ & $38.98 \pm 5.70$ & 0.888 \\
\hline $7^{\text {th }}$ day & $37.92 \pm 4.93$ & $36.64 \pm 6.65$ & 0.514 & $37.87 \pm 4.97$ & $36.57 \pm 6.13$ & 0.371 \\
\hline CVP 1 ${ }^{\text {st }}$ day & $10.50 \pm 3.4$ & $12.02 \pm 5.188$ & 0.191 & $9.47 \pm 2.5$ & $10.43 \pm 2.59$ & 0.153 \\
\hline $4^{\text {rd }}$ day & $9.73 \pm 2.64$ & $11.03 \pm 3.47$ & 0.108 & $19.43 \pm 2.86$ & $10.5 \pm 2.59$ & 0.117 \\
\hline
\end{tabular}




\begin{tabular}{|c|c|c|c|c|c|c|}
\hline \multirow{2}{*}{$\begin{array}{c}\text { Hemodynamic } \\
\text { parameters }\end{array}$} & \multicolumn{2}{|c|}{ (base line) before CPT } & \multicolumn{3}{|c|}{ After $2 \mathrm{~h}$ of CPT } & \multirow[b]{2}{*}{ p value } \\
\hline & $\begin{array}{l}\text { Study } \\
\text { group }\end{array}$ & $\begin{array}{l}\text { Control } \\
\text { group }\end{array}$ & p value & $\begin{array}{l}\text { study } \\
\text { group }\end{array}$ & control group & \\
\hline $7^{\text {th }}$ day & $8.86 \pm 2.60$ & $9.6 \pm 1.71$ & 0.196 & $8.9 \pm 2.41$ & $9.75 \pm 1.70$ & 0.124 \\
\hline
\end{tabular}

n.s $p>0.05$ no significance $* p<0.05$ significance $* * p<0.001$ moderate significance $* * * p<0.000$ high significance

Table (6): Comparison between both study \& control group in relation to respiratory assessment for (Breathing sounds).

\begin{tabular}{|c|c|c|c|c|c|c|c|c|c|c|c|c|c|}
\hline \multirow{3}{*}{$\begin{array}{c}\text { Breath } \\
\text { sound } \\
\text { Days }\end{array}$} & \multicolumn{4}{|c|}{ Normal } & \multicolumn{4}{|c|}{ Crackles } & \multicolumn{4}{|c|}{ Wheezing } & \multirow{3}{*}{$P$ value } \\
\hline & \multicolumn{2}{|c|}{$\begin{array}{l}\text { Study } \\
\text { group }\end{array}$} & \multicolumn{2}{|c|}{$\begin{array}{c}\text { Control } \\
\text { group }\end{array}$} & \multicolumn{2}{|c|}{$\begin{array}{l}\text { Study } \\
\text { group }\end{array}$} & \multicolumn{2}{|c|}{$\begin{array}{l}\text { Control } \\
\text { group }\end{array}$} & \multicolumn{2}{|c|}{$\begin{array}{l}\text { Study } \\
\text { group }\end{array}$} & \multicolumn{2}{|c|}{$\begin{array}{l}\text { Control } \\
\text { group }\end{array}$} & \\
\hline & $\mathbf{N}$ & $\%$ & $\mathbf{N}$ & $\%$ & $\mathbf{N}$ & $\%$ & $\mathbf{N}$ & $\%$ & $\mathbf{N}$ & $\%$ & $\mathbf{N}$ & $\%$ & \\
\hline $1^{\text {st }}$ day & 7 & 23.33 & 0 & 0 & 4 & 13.33 & 9 & 30.0 & 19 & 63.7 & 21 & 70.0 & $0.011 *$ \\
\hline $4^{\text {rd }}$ day & 6 & 20.0 & 1 & 3.3 & 6 & 20.0 & 7 & 23.33 & 18 & 60.0 & 22 & 73.33 & 0.132 \\
\hline $7^{\text {th }}$ day & 12 & 40.0 & 2 & 6.7 & 6 & 20.0 & 5 & 16.6 & 12 & 40.0 & 23 & 76.7 & $0.005 * *$ \\
\hline
\end{tabular}

n.s $p>0.05$ no significance $* p<0.05$ significance $* * p<0.001$ moderate significance $* * * p<0.000$ high significance

Table (7): Comparison between the study \& control group as regard respiratory rate\& mean values in both groups.

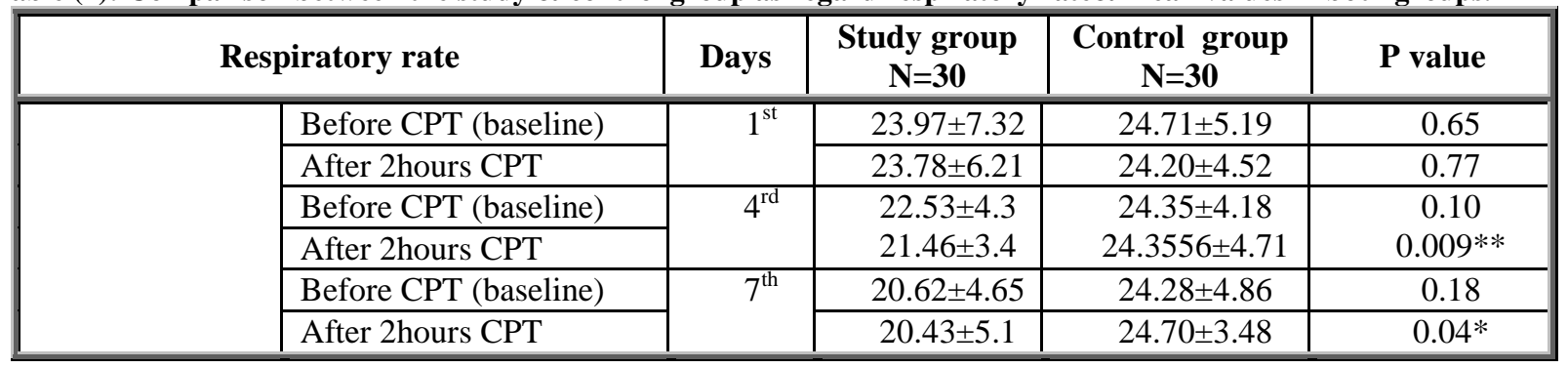

n.s $p>0.05$ no significance $* p<0.05$ significance $* * p<0.001$ moderate significance

$* * * p<0.000$ high significance

Table (8): Comparison between the study and control groups in relation to arterial blood gases \& mean values in both groups.

\begin{tabular}{|c|c|c|c|c|c|c|}
\hline \multirow[b]{2}{*}{ Arterial blood gases } & \multicolumn{2}{|c|}{ (base line) before CPT } & \multirow[b]{2}{*}{ p value } & \multicolumn{2}{|c|}{ After $2 \mathrm{~h}$ of CPT } & \multirow[b]{2}{*}{ p value } \\
\hline & $\begin{array}{l}\text { Study } \\
\text { group }\end{array}$ & $\begin{array}{l}\text { Control } \\
\text { group }\end{array}$ & & $\begin{array}{l}\text { study } \\
\text { group }\end{array}$ & $\begin{array}{l}\text { control } \\
\text { group }\end{array}$ & \\
\hline $\mathrm{pH} 1^{\text {st }}$ day & $7.38 \pm 0.07$ & $7.38 \pm 0.08$ & 0.942 & $7.39 \pm 0.06$ & $7.39 \pm 0.08$ & 0.916 \\
\hline $4^{\text {rd }}$ day & $7.40 \pm 0.63$ & $7.36 \pm 0.05$ & $0.03 *$ & $7.40 \pm 0.05$ & $7.36 \pm 0.62$ & $0.02 *$ \\
\hline $7^{\text {th }}$ day & $7.40 \pm 0.06$ & $7.36 \pm 0.06$ & 0.225 & $7.39 \pm 0.06$ & $7.37 \pm 0.06$ & 0.062 \\
\hline $\mathrm{PaO} 21^{\text {st }}$ day & $74.27 \pm 16.49$ & $70.33 \pm 16.79$ & 0.363 & $79.34 \pm 11.98$ & $79.94 \pm 8.88$ & 0.825 \\
\hline $4^{\text {rd }}$ day & $79.71 \pm 11.82$ & $77.31 \pm 8.63$ & 0.872 & $82.89 \pm 9.88$ & $81.97 \pm 8.59$ & 0.733 \\
\hline $7^{\text {th }}$ day & $82.89 \pm 9.88$ & $81.97 \pm 8.59$ & 0.702 & $83.81 \pm 9.79$ & $83.59 \pm 8.11$ & 0.927 \\
\hline $\mathrm{SaO} 21^{\text {st }}$ day & $90.83 \pm 5.36$ & $90.73 \pm 7.19$ & 0.953 & $91.881 \pm 5.83$ & $91.22 \pm 6.86$ & 0.690 \\
\hline $4^{\text {rd }}$ day & $93.23 \pm 3.47$ & $93.45 \pm 3.08$ & 0.794 & $93.61 \pm 3.19$ & $93.16 \pm 2.99$ & 0.579 \\
\hline $7^{\text {th }}$ day & $93.57 \pm 5.76$ & $93.33 \pm 3.09$ & 0.838 & $93.85 \pm 0.29$ & $94.08 \pm 3.39$ & 0.788 \\
\hline $\mathrm{PaCO} 21^{\text {st }}$ day & $55.99 \pm 15.84$ & $54.49 \pm 15.45$ & 0.713 & $74.27 \pm 10.09$ & $75.01 \pm 6.99$ & 0.740 \\
\hline $4^{\text {rd }}$ day & $77.71 \pm 11.82$ & $78.16 \pm 9.26$ & 0.872 & $78.39 \pm 11.99$ & $79.31 \pm 8.63$ & 0.733 \\
\hline $7^{\text {th }}$ day & $48.07 \pm 13.22$ & $42.71 \pm 9.76$ & 0.079 & $46.59 \pm 12.68$ & $41.39 \pm 8.66$ & 0.069 \\
\hline HcO3 $1^{\text {st }}$ day & $32.59 \pm 6.79$ & $30.97 \pm 4.92$ & 0.294 & $33.22 \pm 6.19$ & $31.28 \pm 4.59$ & 0.173 \\
\hline $4^{\text {rd }}$ day & $50.79 \pm 13.41$ & $48.32 \pm 13.09$ & 0.470 & $78.39 \pm 11.99$ & $79.31 \pm 8.63$ & 0.733 \\
\hline $7^{\text {th }}$ day & $33.69 \pm 7.89$ & $30.89 \pm 3.59$ & 0.083 & $34.42 \pm 6.57$ & $31.22 \pm 4.71$ & $0.034 *$ \\
\hline
\end{tabular}

n.s $p>0.05$ no significance $* p<0.05$ significance $* * p<0.001$ moderate significance oxygen saturation (SOa $\left.{ }_{2}\right)$ $* * * p<0.000$ high significance level of blood $(\mathrm{PH})$, partial pressure of arterial oxygen $\left(\mathrm{PaO}_{2,}\right)$ partial pressure of arterial carbon dioxide $\left(\mathrm{PaCo}_{2}\right)$, level of bicarbonate in blood $\left(\mathrm{HCO}_{3}\right)$ 
Table (1): Showed the mean value of age was $(53.63 \pm 7.425)$ for the study group and $(51.93 \pm 8.034)$ for the control group respectively. There was no statistical differences between both groups $p \geq 0.05$ Table (2): Showed data for patients complain with no significance difference in study and control groups ( $\mathrm{P}$ value $=0.603)$ there were $(26.7 \% \mathrm{Vs} 33.3 \%)$ in study and control groups respectively had productive cough, while $(16.7 \%$ Vs $26.7 \%)$ in study and control groups respectively had chest wheezing \&crepitation. while $(30.0 \% \mathrm{Vs} 23.3 \%)$ in study and control groups respectively had fever \& chest pain while $(26.7 \%$ Vs $16.7 \%)$ in study and control groups respectively had Dyspnea\& hypoxemia.

As regard presence of any chronic disease where (23.3\% Vs 53.3)in study and control groups respectively had history of hepatic disease with significance difference $(\mathrm{P}$ value $=0.016 *)$ Where (3.3\%) of study and control groups were history of stroke, $(16.7 \%$ Vs $20.0 \%)$ in study and control groups respectively had history of ischemic disorder. Where $(20.0 \%)$ of study and control groups were history of hypertensive. $(40.0 \% \mathrm{Vs} 33.3 \%)$ in study and control groups respectively had history of diabetes mellitus. Also regarding presence of past medical history It was noticed that moderate significance difference of both groups it was $(40.0 \%)$ in study $(66.7 \%)$ and in control groups $\mathrm{p} \leq 0.05$.

Table (3): In relation to hematological laboratory investigation the result revealed that no significance difference regarding complete blood picture there was at $1^{\text {st }}, 4 \mathrm{rd} \& 7^{\text {th }}$ days between both groups $\mathrm{p} \geq 0.05$. While regarding prothrombin time there were no significance differences at $1^{\text {st }}, 4 \mathrm{rd} \& 7^{\text {th }}$ days between both groups $\mathrm{p} \geq 0.05$

Table(4): Showed medication received in study \& control groups with no significance difference about anticoagulant, antihypertensive, osmotic diuretic, antibiotic, antipyretic and inhalation $p \geq 0.05$. While there were significance difference $(\mathrm{P}=0.03)$ about bronchodilators in first day. And moderate difference regard corticosteroid therapy in first day $(\mathrm{P}=0.02)$.

Table (5): As regard to temperature $\left(C^{0}\right)$ it can observe that the mean value were no statistically significant in study group compared to control group after 2hours of CPT in (1) \& (4) days $p \geq 0.05$. While there were highly significant difference before \& after 2 hours of CPT between both groups $(\mathrm{P}=0.000)$. As regard to central venous pressure it noticed there is no significant difference between both groups before \& after 2 hours of CPT $\mathrm{p} \geq 0.05$. So that can observed the whole study sample was normotensive. As regard to systolic and diastolic blood pressure (BP) it can observed there is no significant difference after 2 hours of CPT between both groups $\mathrm{p} \geq 0.05$ and the whole mean study sample was normotensive.

As regard to mean arterial pressure it can observe that no significant difference before \& after 2 hours of CPT between both groups $\mathrm{p} \geq 0.05$.

As regarding to the pulse it can observe that $(98.33 \pm 15.87)$ in study group and $(98.34 \pm 12.11)$ in control group before \& after 2 hours of CPT in 1 days was no significant different ( $\mathrm{p} \geq 0.05)$. While in 4 days can reported that highly significant $(98.62 \pm 13.87)$ in study group comparing to control group $(101.18 \pm 12.48)$ after 2 hours of CPT $(p=0.008)$. While in 7 days present highly significant $(\mathrm{p}=0.002)$ which the study group $(95.09 \pm 12.45)$ compared to control group $(106.64 \pm 12.86)$ before $\&$ after CPT.

Table (6): Showed comparison between both groups in relation to breath sound. The result revealed that significant difference $(\mathrm{p}=0.01)$ in normal breath sound $(23.33 \%)$ in first day. While in seven days $(40.0 \%$ vs. $6.7 \%)$ of the study group comparing to control group had normal sound with highly significant difference $(\mathrm{p}=0.005)$. As regarding abnormal breath sound as (Crackles, wheezing) findings that $(13.33 \%)$ in study group compared to $(30.0 \%)$ in the control group and $(40.0 \%)$ in study group compared to $(76.7 \%)$ in the control group with highly significant different $(\mathrm{p}=0.005)$.

Table (7): Showed comparison between both study $\&$ control groups as regard respiratory rate which the results revealed that no significant difference ( $p \geq 0.05)$ in study \& control groups in first day. While in four days mean value was higher among study group comparing to control group after 2 hours of CPT $(\mathrm{P}<0.009 * *)$ and $)$ in seven days observed highly significant difference between study and control groups with $(\mathrm{p}=0.004)$.

Table (8): Showed comparison between both study $\&$ control groups in relation of arterial blood gases it revealed there were no significant difference between study and control groups before \&after CPT in first day. While there were significant difference between study and control groups before \&after CPT in four and seven day with $(\mathrm{P}=0.03)$.

\section{Discussion}

Based on the results of the present study, the patient's socio demographic data between the study and control groups were comparable and no significant differences were founded. These finding was agreeing with the study done by (Abd- Elwanees et al., 2014) who reported that there were no any significance differences in the age, gender, and other demographic data.

As regard age and gender, the present study revealed that no statistically significant difference in both the 
study and control groups. This finding came in line with the study done by Kaiqin, (2018) who conduct his study on 289 cases that age distribution, was no statistical significance between male patients and female patients, whom were mainly in 70-89 years old.

The present study found that there was no significant statistical difference between the study and control groups regarding medical data except hepatic disease which revealed that present significant difference between both groups This came in contrast with the study done by (Alaa Eldin \& Mohamed, 2017) who showed that statistically significant differences for medical data in both groups and also in regard chest wheezing and dyspnea.

Regarding ischemic heart disease the current study results revealed that there was no significance difference between both groups regard IHD. This finding came in line with the study done by Tadros, (2017) who showed that there's no statistically significant difference observed regarding possible acute ischemic changes.

Mehta, (2018) found that not any correlation between following comorbidities and mortality: systemic hypertension, diabetes mellitus, coronary artery disease, peripheral vascular disease, congestive heart failure, chronic kidney disease, chronic liver disease, and pulmonary artery hypertension.

Regarding investigations The present study revealed that, no significant difference regarding WBC between the two groups this is in contrast with study done by Salahuddin et al., (2010) who revealed that WBC statistically higher significant difference.

As regard hemoglobin and total leukocyte count level there were no significant difference in both groups. This is in line with (Ittyachen et al., 2016) with no significant difference in hemoglobin and total leukocyte count level in both groups, total count was raised which indicates that infection is the most common cause of exacerbation in patients of COPD.

Philip, (2012) reported that hemodynamic monitoring is central necessarily forms for a major aspect of intensive care nursing to ICU patients.

As regard to body temperature $\mathrm{C}$ the findings of current study revealed that there were highly statistical significance difference found after $2 \mathrm{~h}$ chest physiotherapy between two groups in seven day and mean score in the control group was moderate higher than in study group.

Rosdal, (2012) mentioned that the critical care nurse should regular monitor measurement of vital signs includes obtaining temperature, blood pressure(BP), pulse rate $(\mathrm{P})$ which provide a picture of how much work the patient must do to maintain stable hemodynamic parameters.
The current study revealed that highly statistical significant difference in four and seven day after CPT in the mean heart of study compared to control group. While in first day after CPT the heart rate within normal in both groups. This improvement of hemodynamic could be explained by the improvement in lung mechanics as well as parameters of the performing CPT that suppress abnormally elevated symptomatic activity this finding is in contrast with the study done by (Costa et al., 2011) who finding that there were no significant differences between the two groups regarding the hemodynamic parameters (HR and MAP).

As regard blood pressure (BP) mean value observed no significant difference after $2 \mathrm{~h}$ of CPT between both groups regarding SBP \&DBP and mean blood pressure the whole study sample was normotensive. This came in contrast with the study done by (Abdel Aziz et al., 2016) mentioned that heart rate was significantly higher in the failure group and both SBP and DBP were significantly lower.

The current study revealed that the majority of study group had normal breath sound compared with control group \& the result revealed that abnormal breath sound as Crackles and wheezing findings highly significance difference in study group compared to control group.

The result was supported by (Wiegand et al., 2010) who mentioned that abnormal breath sounds might indicate presence of chest infection need more assessment and intervention.

In the present study the assessment of respiratory system showed that there was a highly significance of crackles and wheezing on study group versus control group. While in seven day there was a highly significance difference of crackles and wheezing on seven day on study group versus control group after 2 hours CPT (Park et al., 2009) mentioned that CPT is used to mobilize and remove secretions in airways in order to improve lung function and facilitate gas exchange.

The present study demonstrate that there were no significant statistical differences regarding ABGs expect a marked improvement in arterial blood gas exchange reflect by significant statistical difference was found between the both groups after 2 hours CPT in mean $\mathrm{PH}$ in $4^{\text {th }}$ day, $\mathrm{HCO} 3$ at $7^{\text {th }}$ days This improvement in $\mathrm{ABG}$ parameters when compared to baseline value by chest physiotherapy which lead to redistribution of blood flow, reducing hypoxic vasoconstriction and better secretion removal.

In this respect (Abd- Elwanees et al., 2014) documented that there was no significant difference in ABGs between both groups on admission. In 
relation to the mean value of $\mathrm{PaCO} 2$ among the study and control groups, the values declined on the both groups from respiratory acidosis to normal acidity. Also the mean value of $\mathrm{PaO} 2$ was relatively improved among patients in the $\mathrm{CP}$ and non $\mathrm{CP}$ groups from admission to discharge. Kaplow, (2010) mentioned that $\mathrm{ABGs}$ is used to determine level of $\mathrm{O} 2, \mathrm{Co} 2$ and $\mathrm{PH}$ to identify acid base disturbances, hypoxemia and monitor any change in respiratory parameters Ware, (2010) added that ABGs improve with the increase in the frequency of number of CPT.

\section{Conclusion}

Based on the findings of the present study, it can be concluded that nursing care for acute exacerbation of chronic obstructive disease patients significantly improve respiratory parameters.

\section{Recommendations}

- Implementing nursing management after acute exacerbation of chronic obstructive pulmonary and improve respiratory parameters.

- Develop educational program for nursing staff caring for patient with acute exacerbation of chronic obstructive pulmonary disease.

- Health professional can utilize such information in the provision of care for acute exacerbation of COPD patient's in the future.

- Chest physiotherapy must be applied as a routine care for cases of acute exacerbation COPD admitted to chest care unit to improve the patient's outcomes.

\section{References}

1. Abdel Aziz, Ali O., (2016): MD, Department of Chest Diseases, Faculty of Medicine, Minia University, Minia, 61519, Egypt; Tel: 0862333196; fax: +20 (86) 234 2601; e-mail: info@minia.edu.eg Accepted 28 November 2016

2. Abd-Elwanees A., El-Soussi A., Othman S., \& Ali R., (2014): Effect of Implementing Clinical pathway on the Clinical Outcomes of patients with Acute Exacerbation of Chronic Obstructive Pulmonary Disease, International Journal of Nursing Science, vol. (4), No. (1):110.

3. Alaa Eldin Metwally \& Mohamed Elgazzar, (2017): Assistant Professor of Chest Disease and Tuberculosis, Faculty of Medicine, Zagazig University, e-mail: alaametwaly72@yahoo.com Received 24 October 2017 Accepted 6 August 2017.
4. Clinical Guideline, (2010): Chronic obstructive pulmonary disease NICE Clinical Guideline June View this article online at: patient.info/doctor/chronic-obstructivepulmonary- disease-pro

5. Costa R., Spinazzola, F., Cipriani, G., Ferrone, O., Festa, A., Arcangeli, A., (2011): physiologic comparison of proportional assist ventilation with load-adjustable gain factors (PAV+) versus pressure support ventilation (PSV), Intensive Care Med. 37 (9) (2011) 14941500,2010;25:25-272018;7:77-82.

6. Dheeraj Gupta1, Jindal Ritesh Agarwal1, Ashutosh Nath Aggarwal1, V., (2014): Maturu1, Sahajal Dhooria1, K.T. Prasad1, Yenge1, Behera1 and S.K. for the COPD Guidelines Working Group The Indian Journal of Chest Diseases \& Allied Sciences Vol.56.

7. Global Initiative for Chronic Obstructive Lung Disease, (2018): Global strategy for the diagnosis, management and prevention of chronic obstructive pulmonary disease; 2018.

8. Holland A., (2014): Physiotherapy management of acute exacerbations of chronic obstructive pulmonary disease. Journal of Physiotherapy 60: 181-188] 2014 Australian Physiotherapy Association. Published by Elsevier B.V.

9. Ittyachen A., Krishnamoorthy S., Bhatt A., Abdulla S., Roy J., Sugathan M., (2016): Predictors of outcome in patients admitted with acute exacerbation of chronic obstructive pulmonary disease in a rural Tertiary Care Center. J Family Med Prim Care 2016;5:411-5.

10. Kaiqin Tian, (2018): Department of pharmacy, The First Affiliated Hospital of Zhengzhou University, Zhengzhou City, 450000, Henan Province, Chin Corresponding author email: Published Online: 10 January 2019.

11. Kochanek K., Murphy S., Xu J., Arias E., (2014): Mortality in the United States 2013. NCHS Data Brief.; (178):1-8.

12. Kochanek, K., Murphy, S., Miniño, A., \& Kung, H., (2011): Deaths: final data for 2009. Natl Vital Stat Rep 60: 3-11.

13. Mehta, Asmita, M., (2018): Clinical Professor, Pulmonary Medicine, AmritaInstitute of Medical Sciences, The Egyptian Journal of Chest Diseases and Tuberculosis 2018, 67:361367.

14. Park K., Oh Chang H., Sheen S., Choi J., (2009): Acute hemodynamic effects of recruitment maneuvers in patients with acute respiratory distress syndrome. J Intensive care Med, 24:476-82 
15. Philip Woodrow, (2012): intensive care nursing Afram work for practice,chapter (7) acute respiratory distress syndrome $3^{\text {rd }}$ edition by Elsevier ,londne \& new York p.p 270-275.

16. Roberta Kaplow, Sonya R., Hardin, (2010): critical care nursing ;synergy for optional outcomes, chapter 21 , respiratory monitoring 475-480.

17. Roberts, C., Lowe, Y., Kelly, C., Bucknall, M., (2017): on behalf of the Audit Subcommittee of the Standards of Care Committee of the British Thoracic Society and the Clinical Effectiveness and Evaluation Unit at the Royal College of Physicians

18. Rosdal, Carolin, Mary T., Kowalski (2012): text book of basic nursing chapter 86 (respiratory disorder) $10^{\text {th }}$ edition phlideliphia, lipincott Williams\&Willkins.p.p1409-1429.

19. Salahuddin N., Irfan M., Khan S., Naeem M., Haque A., Husain S., (2010): Variables predictive of outcome in patients with acute hypercapneic respiratory failure treated with noninvasive ventilation. J Pak Med Assoc 2010; 60:13-17.

20. Tadross B., (2017): MSc, 128Taowniat Smouha, Smouha, Alexandria, Egypt; Tel: 20101280509; E-mail: dr_bisho@ hotmail.com Received 17 January 2017 Accepted 26 February 2017Research and Opinion in Anesthesia \&Intensive Care 2017, 4:54-58

21. Ware L., (2010): pathophysiological of acute lung injury and the acute respiratory distress syndrome. Respir crit care Med ,27(4):337-349.

22. Weigand Brill Jadwiga A., Wedzicha (2010): Airway Disease Section, National Heart and Lung Institute, Imperial College, London, UK Correspondence: Simon EB rill Airway Disease Section, National Heart and Lung Institute, International Journal of COPD 2014:9 12411252.

23. Williams V., Bruton A., Ellis-Hill C., McPherson K., (2011): The importance of movement for people living with chronic obstructive pulmonary disease. Qual Health Res. 2011;21(9):1239-1248.

24. World Health Organization WHO, (2013): Chronic obstructive pulmonary disease (COPD), Fact sheet No. 315, Reviewed October 2013. 